\title{
Optical Oxygen Sensors With Improved Lifetime Incorporating Titania Beads and Polydimethylsiloxane Coatings
}

\author{
Emily CIRULNICK, Haopeng ZHANG, and David KLOTZKIN*
}

Electrical and Computer Engineering Department, Watson School of Engineering, Binghamton University, Binghamton NY 13902-6000, The United States of America

${ }^{*}$ Corresponding author: David KLOTZKIN_Ｅ-mail: klotzkin@binghamton.edu

\begin{abstract}
The use of optical sensors for oxygen measurement is becoming more important because of their capability for low-cost and direct measurement, but as yet, little has been reported about their long-term performance. Phosphorescent sensors based on platinum octaethylporphyrin (PtOEP) embedded in polymer matrices tend to degrade with time. To reduce the rate of degradation, sensor films were fabricated and then coated with a layer of polydimethylsiloxane (PDMS) and tested in a six-month study. The PDMS-coated sensors showed an average degradation rate of $\sim 0.073 \% /$ day, compared to $\sim 0.18 \%$ /day for uncoated sensors. Titania beads were also incorporated into the films to increase light scattering and improve the response; these beads compensated to some degree for the absorption due to the PDMS films. The films with titania beads improved the response significantly (about 40\%) compared to the films without titania beads. Incorporation of titania beads also moderately improved the aging characteristics.
\end{abstract}

Keywords: Fluorescence; optical oxygen sensor; photoluminescence; aging

Citation: Emily CIRULNICK, Haopeng ZHANG, and David KLOTZKIN, "Optical Oxygen Sensors With Improved Lifetime Incorporating Titania Beads and Polydimethylsiloxane Coatings,” Photonic Sensors, 2022, 12(1): 68-73.

\section{Introduction}

The significance of oxygen in different areas, such as clinical diagnosis, bioengineering research, and environmental monitoring, has led to high interest in sensors for measuring oxygen concentration. Much research has been focused on optical methods of measuring oxygen, typically based on quenching of luminescence emitted by oxygen-sensitive dyes. Compared to electrochemical Clark electrode sensors, luminescence sensors offer quick response, no oxygen consumption, high sensitivity, and robustness $[1,2]$. These sensors can also be integrated into optical fiber facets and interrogated remotely [3]. The issue until recently had been the need for large and expensive interface components, such as spectrophotometers; however, optical interrogators have been miniaturized in recent years, leading to compact fluorescent measurement and inexpensive packaging.

However, fluorescent sensors tend to age with exposure to the environment, and their response degrades significantly over a time scale of months. Degradation in performance has been reported both in water [4] and in air [5, 6]. This degradation limits the operating and shelf time of these sensors and is a practical limitation to their adoption. Absorption of water into the film is believed to block $\mathrm{O}_{2}$ access to

Received: 12 June 2020 / Revised: 8 September 2020

(C) The Author(s) 2020. This article is published with open access at Springerlink.com DOI: $10.1007 / \mathrm{s} 13320-020-0608-\mathrm{z}$

Article type: Regular 
the dye sites by reducing permeability of the matrix [7], and sensors generally demonstrate reduced response at higher humidity [8]. While much work has been reported on these optical sensors, relatively little has focused on their aging characteristics.

In this paper, polydimethylsiloxane (PDMS) coatings were applied to sensor films composed of a polymer and an oxygen-sensitive chemical platinum-porphyrin (PtOEP). PtOEP exhibits $\mathrm{O}_{2}$ dependent emission quenching, with both the intensity and the phosphorescent lifetime being a strong function of $\mathrm{O}_{2}$ concentration. Measurements of both have been used to quantify $\mathrm{O}_{2}$ concentration $[9,10]$. Sensor films were fabricated both with and without a PDMS layer and the response is measured over a period of six months to evaluate the effect of PDMS on aging characteristics. PDMS was $\mathrm{O}_{2}$-permeable and water-impermeable [11] and so was thought to be likely to improve the aging response.

To compensate to some extent for the absorption and scattering of light in the top PDMS layer, and to potentially improve the aging characteristics, some of the sensor films also incorporated titania beads. The beads have been shown to scatter the light and lead to an improved response $[12,13]$. In addition, coatings made with mixtures of PDMS and $\mathrm{TiO}_{2}$ nanoparticles have previously shown improved hydrophobicity [14-16], for potentially better aging response. Sensors both with and without PDMS, also with and without titania beads are tested over a period of six months, and the results are reported.

\section{Methodology}

The oxygen sensor is based on metalloporphyrin lumiphore platinum octaethylporphyrin (PtOEP) encapsulated in ethyl cellulose (EC). The oxygen film was fabricated as follows: $2.2 \mathrm{~g}$ of EC were mixed with $30 \mathrm{~mL}$ of tetrahydrofuran (THF) and the EC was left to dissolve in the THF for 24 hours. $20 \mathrm{mg}$ of PtOEP then were added to the mixture. For those sensors with titania beads, $50 \mathrm{mg}$ of titania beads (Sigma Aldrich 634662, size $<100 \mathrm{~nm}$ ) were also added to the solution. It was then left to dissolve for another 24 hours. The solution was then spin-coated onto a standard $25 \mathrm{~mm}$ by $75 \mathrm{~mm}$ microscope slide at $1800 \mathrm{RPM}$ for one minute. The film was then left to dry for 24 hours. For films with a PDMS top layer, Sylcap ${ }^{\text {TM }} 2840$ S standard elastomer based was mixed with a curing agent in a ratio of $10: 1$, then spin-coated on the film at 500 RPM for 10 seconds followed by 5000 RPM for 30 seconds and left 24 hours- 48 hours to fully cure before use. This process gave a PDMS thickness of about $13 \mu \mathrm{m}$ [17].

A green light-emitting diode (LED) (Bivar R20GRN-F-0160) with central emission wavelength $530 \mathrm{~nm}$ was used as the excitation light source. The excitation light was linearly polarized by passing through Polarizer 1 (NT45667, Edmund Optics). The randomly polarized red emission of the PtOEP film passed through Polarizer 2. Meanwhile, the polarized excitation light not absorbed by the PtOEP film was blocked by Polarizer 2, as shown in Fig. 1.

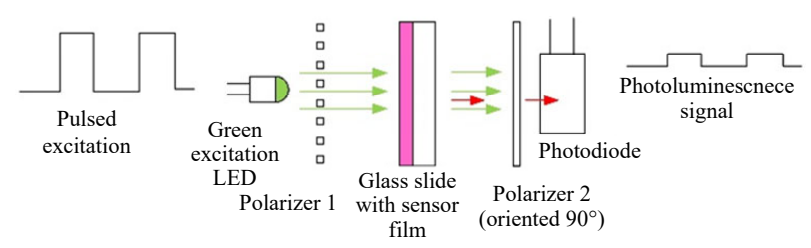

Fig. 1 Schematic diagram of the sensor apparatus. The excitation light is polarized, and then incident on the oxygen-sensitive film, and the majority of excitation light passes through the film is blocked from the detector by the second, orthogonally-oriented polarizer. The emission light, randomly polarized, goes through the polarizer and is detected by the detector. The excitation light is driven pulsed and measured at that frequency to reduce noise.

When cross-polarized, the extinction ratio of excitation light to leakage light was $28 \mathrm{~dB}$ [18]. More details of this polarization-filtered test setup were reported in $[19,20]$. This technique is quite flexible (as it can be used with any fluorescent or phosphorescent dye without change in hardware or need for a color filter) but it does not result in measurement sensitivity as high as color-filter methods. 

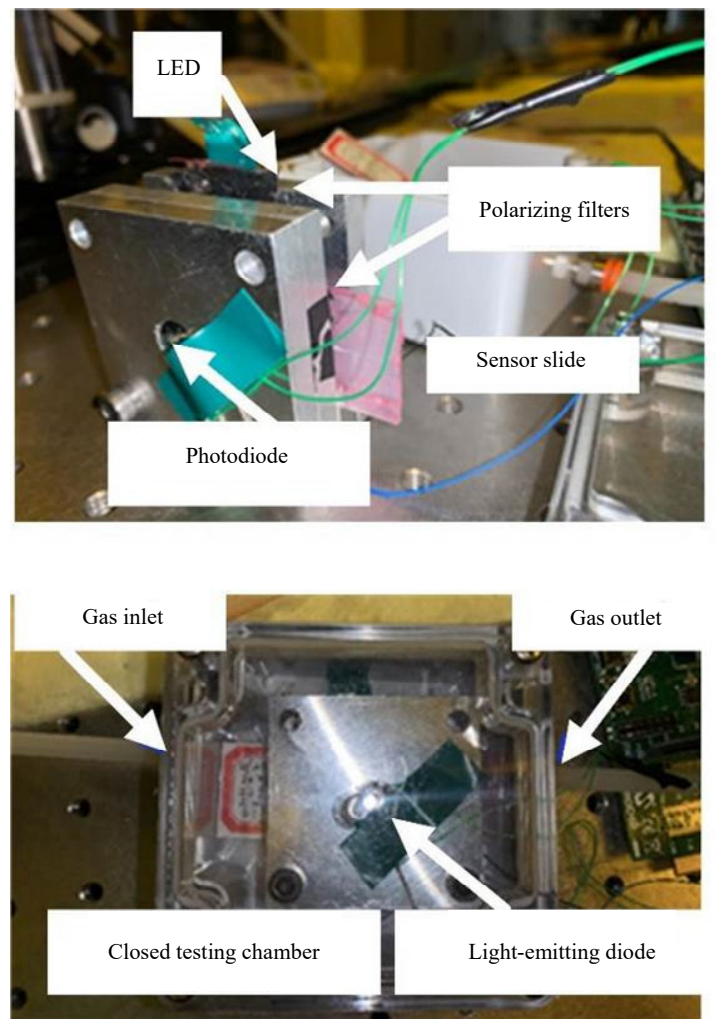

Fig. 2 Sensor structure with glass film slide (top) and sensor box with inlet gas and outlet attachment (bottom) to commercial sensor.

LED was modulated at a convenient moderate frequency $(1 \mathrm{kHz})$ and the response measured at that frequency by a photodiode (Thorlabs FDS1010) in order to reduce the effects of ambient noise. A microprocessor drove the LED and digitized and measured the response from the photodiode. The response time of the system was of the order of a second, likely limited by the time required for gas exchange and similar to what has been reported [21, 22].

The system was tested by putting the sensor, LED, and photodiode in a small box with a gas inlet and outlet. The outlet was connected to a commercial oxygen sensor and the inlet was connected to a Y-junction connected to two, flow-regulated, gas bottles of nitrogen, and oxygen, respectively. Figure 2 shows a picture of the test setup, with the sensor film sandwiched by the light emitting diodes and the photodiode in the gas chamber. By varying the flow rates of the two bottles and monitoring the $\mathrm{O}_{2}$ concentration with the commercial sensor, the concentration of oxygen can be varied between 0 and $100 \%$, and the response plotted vs. concentration.

The measurements were quantified by a digitized pulse height of the filtered fluorescent signal that was reported in arbitrary units. The interrogator measured one hundred individual points and reported the averages to minimize random errors and electronic noise.

The films were tested every week or so over a six-month period. As it was more controllable to set the concentration to $100 \% \mathrm{O}_{2}$, and then $100 \% \mathrm{~N}_{2}$ $\left(0 \% \mathrm{O}_{2}\right)$, than to equilibrate $\mathrm{O}_{2}$ and $\mathrm{N}_{2}$ flow to a stable $50 \%$ concentration, what was recorded was the range of sensor response between $0 \% \mathrm{O}_{2}$ and $100 \% \mathrm{O}_{2}$. This range, recorded in arbitrary units, was normalized to the maximum range observed over the time period to enable direct comparison of the degradation of various devices.

During the course of the six-month study, the sensor films were stored in a lab drawer. The temperature of the lab was set to $70 \mathrm{~F}$ throughout the year with measured humidity varying from $45 \%$ in the winter to up to $75 \%$ or more in the summer.

\section{Results}

Figure 3 shows the response at the end of six-month aging of several representative oxygen sensors, including PDMS-coated with titania beads, PDMS-coated without titania beads, uncoated with titania beads, and uncoated.

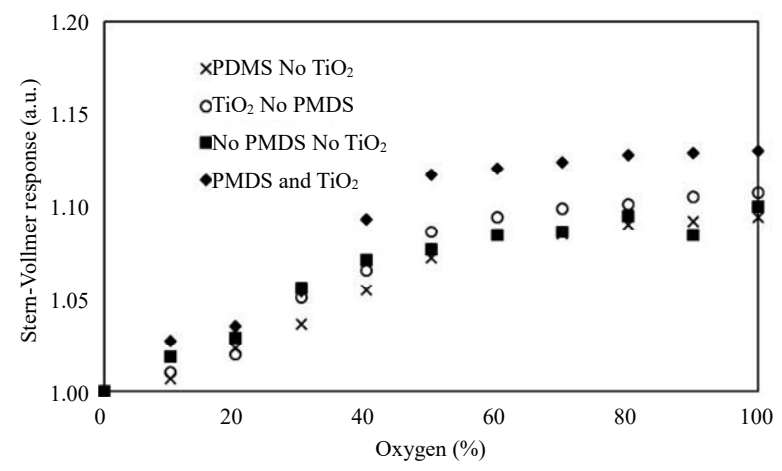

Fig. 3 Stern-Vollmer response of four different sensors, made both with and without PDMS coatings and $\mathrm{TiO}_{2}$ beads. 
The responses are normalized with the Stern-Vollmer relation

$$
\frac{I_{0}}{I}=1+K_{\mathrm{sv}}\left[\mathrm{O}_{2}\right]
$$

where $K_{\mathrm{sv}}$ is the empirical slope, $I$ is the response number reported from the interrogator, and $I_{0}$ is the maximum response with no $\mathrm{O}_{2}$ present. The slope was extracted from the linear region up to $50 \% \mathrm{O}_{2}$ concentration: above $50 \%$, and the response was largely saturated. The linear fits had correlation coefficients to the data of at least $>0.92$ for all the sets of samples. Typical errors for the measurement for a given $\mathrm{O}_{2}$ level were about 0.008 (roughly 4\%, depending on the sensor) based on the standard deviation of measurements taken on different days.

Figure 4 shows the range of response measured between 0 and $100 \% \quad \mathrm{O}_{2}$ normalized to the maximum range observed, plotted against the number of days since the start of the study. To enable quantitative comparison, each set of data was fit to a linear slope to enable comparison of the slopes/decay rate.

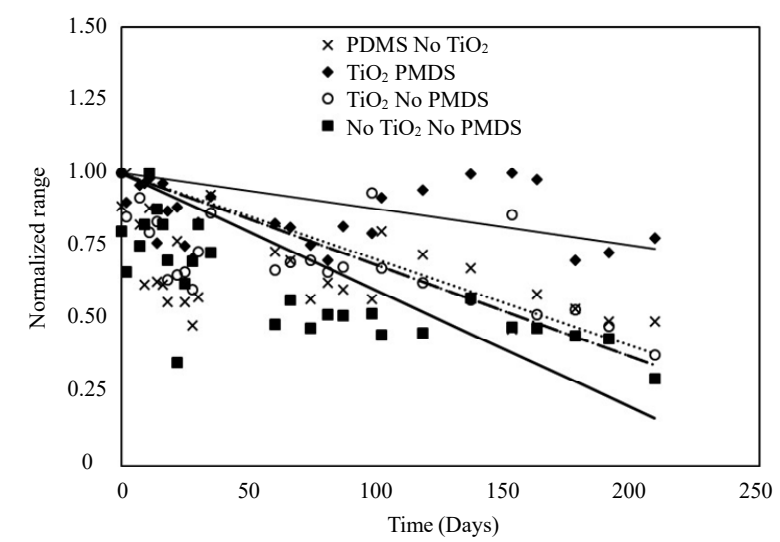

Fig. 4 Range normalized to the maximum range seen over the testing period of the $\mathrm{O}_{2}$ sensor response between 0 and $100 \% \mathrm{O}_{2}$. The trend lines shown (linear fits to the data) are drawn to intercept at 1 and to illustrate the difference in degradation between PDMS coated and non-PDMS coated devices.

Table 1 contains some of the figures of merit of the device, including the Stern-Vollmer slope $K_{\mathrm{sv}}$ (which measures the sensitivity) and the aging seen over our six-month study.

From Table 1 and Fig.4, several trends emerge.
The inclusion of $\mathrm{TiO}_{2}$ increased the sensitivity shown through the increased Stern-Vollmer slope at the end of the testing period. Inclusion of PDMS promoted a reduced decay rate and a longer operating lifetime, though at the expense of sensitivity. The best device showed about $25 \%$ decline over the course of the study.

Table 1 Characteristics of different devices.

\begin{tabular}{ccc}
\hline Device & Aging (\%/day) & Stern-Vollmer slope $K_{\mathrm{sv}}$ \\
\hline No PDMS No $\mathrm{TiO}_{2}$ & $-0.206 \%$ & 0.163 \\
$\mathrm{PDMS} \mathrm{No} \mathrm{TiO}_{2}$ & $-0.115 \%$ & 0.148 \\
$\mathrm{No} \mathrm{PDMS} \mathrm{TiO}_{2}$ & $-0.155 \%$ & 0.180 \\
$\mathrm{PDMS} \mathrm{TiO}_{2}$ & $-0.032 \%$ & 0.250 \\
\hline
\end{tabular}

There is significant noise in the data of Fig. 3, attributed to both the mechanics of the setup, which leads to variations about exactly which spot on the slide was illuminated during the test, and potentially to the variation in conditions (particularly humidity) over the many days in which data was taken.

To evaluate whether the measured difference in slopes was significant, two different statistical analyses were performed. First, an analysis of covariance (ANCOVA) was performed to test the statistical significance of the different aging rates (in \%/day) shown in Table 1 . The most definitive conclusion was that the device containing PDMS and $\mathrm{TiO}_{2}$ was significantly better than the devices without PDMS at the 1\% level. Distinctions between other devices were less clear: the device with just PDMS was significantly better than the devices without $\mathrm{TiO}_{2}$ or PDMS at the $10 \%$ level, but no statistically significant difference between the device with just PDMS and the device with just $\mathrm{TiO}_{2}$ was seen.

In the second analysis, the aging slope was analyzed with a multiple regression test with the two different levels of PDMS or not, and $\mathrm{TiO}_{2}$ or not, taken as inputs. The presence of PDMS was statistically significant to aging rate at the $10 \%$ level, indicating that it did improve the degradation rate. The presence of $\mathrm{TiO}_{2}$ was significant at the $15 \%$ 
level, which was not as definitive but suggested that it was also beneficial. A larger study would be needed for a more definitive answer.

\section{Conclusions}

Coating the devices with PDMS served to reduce their degradation significantly compared to the uncoated devices. Based on PDMS being a moisture barrier, this suggested that a part of the degradation was associated with water vapor. Inclusion of $\mathrm{TiO}_{2}$ increased the numerical range reported by the interrogator, and the Stern-Vollmer slope indicated a more sensitive device. In addition, inclusion of the $\mathrm{TiO}_{2}$ appeared to improve the aging response as well. This was consistent with reports of hydrophobic coatings developed with $\mathrm{TiO}_{2}$ and PDMS.

Further study would be beneficial to strengthen this conclusion, as the data showed significant variation over the course of the study.

Open Access This article is distributed under the terms of the Creative Commons Attribution 4.0 International License (http://creativecommons.org/licenses/by/4.0/), which permits unrestricted use, distribution, and reproduction in any medium, provided you give appropriate credit to the original author(s) and the source, provide a link to the Creative Commons license, and indicate if changes were made.

\section{Acknowledgment}

We gratefully acknowledge the support of Air Liquide, and Thierry Boulanger, in this research.

\section{References}

[1] J. Bacon and J. N. Demas, "Determination of oxygen concentrations by luminescence quenching of a polymer-immobilized transition-metal complex," Analytical Chemistry, 1987, 59(23): 2780-2785.

[2] T. Yeh, C. Chu, and Y. Lo, "Highly sensitive optical fiber oxygen sensor using Pt (II) complex embedded in sol-gel matrices," Sensors and Actuators B: Chemical, 2006, 119(2): 701-707.

[3] C. Chu, Y Lo, and T. Sung, "Review on recent developments of fluorescent oxygen and carbon dioxide optical fiber sensors," Photonic Sensors, 2011, 1(3): 234-250.
[4] Y. Cai, A. Smith, J. Shinar, and R. Shinar, "Data analysis and aging in phosphorescent oxygen-based sensors," Sensors and Actuators B: Chemical, 2010, 146(1): 14-22.

[5] M. Ratterman, "Multi-analyte lab on a chip detection utilizing optical and electro-chemical methods," $\mathrm{PhD}$ dissertation, University of Cincinnati, Cincinnati, Ohio, America, 2015.

[6] C. Chu and T. Lin, "A new portable optical sensor for dual sensing of temperature and oxygen," Sensors and Actuators B: Chemical, 2014, 202: 508-515.

[7] P. Douglas and K. Eaton, "Response characteristics of thin film oxygen sensors, $\mathrm{Pt}$ and $\mathrm{Pd}$ octaethylporphyrins in polymer films," Sensors and Actuators B: Chemical, 2002, 82(2-3): 200-208.

[8] W. Cui, R. Liu, E. Manna, J. Park, F. Fungura, J. Shinar, et al., "Oxygen and relative humidity monitoring with films tailored for enhanced photoluminescence," Analytica Chimica Acta, 2015, 853: 563-571.

[9] S. Ji, W. Wu, Y. Wu, T. Zhao, F. Zhou, Y. Yang, et al., "Real-time monitoring of luminescent lifetime changes of PtOEP oxygen sensing film with LED/photodiode-based time-domain lifetime device," Analyst, 2009, 134(5): 958-965.

[10] A. Smith, Y. Cai, S. Vengasandra, R. Shinar, and J. Shinar, "Advances toward commercialization of a new generation of low cost (O) LED-based dissolved oxygen and bioanalyte monitors," Organic Semiconductors in Sensors and Bioelectronics III, 2010, 7779: 77790.

[11] J. McDonald, J. Cooper, D. Duffy, J. Anderson, D. Chiu, H. Wu, et al., "Fabrication of microfluidic systems in poly (dimethylsiloxane)," Electrophoresis: An International Journal, 2000, 21(1): 27-40.

[12] Y. Cai, R. Shinar, Z. Zhou, and J. Shinar, "OLED-based sensor array for simultaneous monitoring of multiple analytes," Organic-Based Chemical and Biological Sensors, 2007, 6659: 665907.

[13] Z. Zhou, R. Shinar, A. Allison, and J. Shinar, "Enhanced photoluminescence of oxygen sensing films through doping with high dielectric constant particles," Advanced Functional Materials, 2007, 17(17): 3530-3537.

[14] Y. Qing, C. Yang, Y. Sun, Y. Zheng, X. Wang, Y. Shang, et al., "Facile fabrication of superhydrophobic surfaces with corrosion resistance by nanocomposite coating of $\mathrm{TiO}_{2}$ and polydimethylsiloxane," Colloids and Surfaces A: Physicochemical and Engineering Aspects, 2015, 484: 471-477.

[15] F. Xu, T. Wang, J. Bohling, A. Maurice, H. Chen, L. $\mathrm{Wu}$, et al., "Extended hydrophobicity and self-cleaning performance of waterborne $\mathrm{PDMS} / \mathrm{TiO}_{2}$ nanocomposite coatings under 
accelerated laboratory and outdoor exposure testing," Journal of Coatings Technology and Research, 2018, 15(5): 1025-1034.

[16] S. Yousefi, P. Tabatabaei-Panah, and J. Seyfi, "Emphasizing the role of surface chemistry on hydrophobicity and cell adhesion behavior of polydimethylsiloxane/ $\mathrm{TiO}_{2}$ nanocomposite films," Colloids and Surfaces B: Biointerfaces, 2018, 167: 492-498.

[17] W. Zhang, G. S. Ferguson, and S. Tatic-Lucic, "Elastomer-supported cold welding for room temperature wafer-level bonding," in 17th IEEE International Conference on Micro Electro Mechanical Systems. Maastricht MEMS 2004 Technical Digest, Netherlands, Jan. 25-29, 2004, pp. 741-744.

[18] A. Banerjee, A. Pais, I. Papautsky, and D. Klotzkin, "A polarization isolation method for high-sensitivity, low-cost on-chip fluorescence detection for microfluidic lab-on-a-chip," IEEE Sensors Journal, 2008, 8(5): 621-627.

[19] A. Pais, A. Banerjee, D. Klotzkin, and I Papautsky, "High-sensitivity, disposable lab-on-a-chip with thin-film organic electronics for fluorescence detection," Lab on a Chip, 2008, 8(5): 794-800.

[20]L. Shen, M Ratterman, D. Klotzkin, and I. Papautsky, "A CMOS optical detection system for point-of-use luminescent oxygen sensing," Sensors and actuators B: Chemical, 2011, 155(1): 430-435.

[21] R. Herne, J. Brocas, and E. Donckt, "The response time of optical sensors based on luminescence quenching," Analytica Chimica Acta, 1998, 364(1-3): 131-141.

[22] L. Shen, "Portable multiplexed optical detection for point-of-care," $\mathrm{PhD}$ dissertation, University of Cincinnati, Cincinnati, OH, America, 2013. 